\title{
Nasopharyngeal carcinoma: molecular pathogenesis and therapeutic developments - CORRIGENDUM
}

\section{Qian Tao and Anthony T.C. Chan}

doi: 10.1017/S1462399407000312, Published by Cambridge University Press, 04 May 2007

In the review by Q. Tao and A.T.C. Chan (Ref. 1), two percentages were given incorrectly in the second paragraph of the section 'Histology of NPC', starting on page 4 of the pdf. The first sentence of this paragraph should have read as follows (with the corrected percentages underlined here):

The World Health Organization (WHO) classification system (1978) acknowledges three types of NPC based on the differentiation status of tumour cells: type I, differentiated, keratinising squamous cell carcinoma (75\% of NPCs in North America; 2\% of NPCs in southern China) (Refs 11, 12); type II, nonkeratinising carcinoma (12\% of NPCs in North America; $3 \%$ of NPCs in southern China); and type III, undifferentiated carcinoma (13\% of NPCs in North America; $95 \%$ of NPCs in southern China).

\section{Reference}

1 Tao, Q. and Chan, A.T.C. (2007) Nasopharyngeal carcinoma: molecular pathogenesis and therapeutic developments. Expert Reviews in Molecular Medicine 9, Issue 12, DOI: 10.1017/S1462399407000312 